\title{
Asymmetry of second harmonic generation in magnetic thin films under circular optical excitation
}

\author{
A. Kirilyuk* \\ Research Institute for Materials, University of Nijmegen, Toernooiveld 1, 6525 ED Nijmegen, The Netherlands \\ V. V. Pavlov and R. V. Pisarev \\ A. F. Ioffe Physical Technical Institute of the Russian Academy of Sciences, 194021 St. Petersburg, Russia \\ Th. Rasing \\ Research Institute for Materials, University of Nijmegen, Toernooiveld 1, 6525 ED Nijmegen, The Netherlands
}

(Received 29 July 1999)

\begin{abstract}
We show that right- and left-handed circularly polarized optical excitation can lead to a different intensity of the second harmonic generation in magnetic thin films. This kind of asymmetry arises due to an interference of nonlinear optical waves of crystallographic and/or magnetic origin. In contrast to linear optics, the equivalence between the magnetization reversal and the change of the light helicity can be broken. These conclusions are confirmed by experimental studies of the nonlinear asymmetry in thin films of magnetic garnets.
\end{abstract}

In linear optics, circularly polarized electromagnetic waves with right- and left-handed helicity interact differently with magnetic crystals, leading to a number of physical phenomena in transmission and reflection of light. Physically, this kind of optical asymmetry arises due to the breaking of the time-reversal operation 1 . To illustrate this, consider the induced polarization $\mathbf{P}^{\omega}$ as a function of the electric field of the incident light $\mathbf{E}^{\omega}$ and the sample magnetization $\mathbf{M}$ (either induced or spontaneous). In the electric-dipole approximation we can write $\mathbf{P}^{\omega}$ as

$$
P_{i}^{\omega}=\chi_{i j}^{(1)} E_{j}^{\omega}+i \beta_{i j k}^{(2)} E_{j}^{\omega} M_{k}
$$

The polar tensor $\chi_{i j}^{(1)}$ is allowed in all media and leads to an asymmetry of the interaction of linearly polarized light with crystals, such as linear birefringence and dichroism. The axial tensor $\beta_{i j k}^{(2)}$ is also allowed in all media. Well-known examples of the relevant optical phenomena when the timereversal symmetry is broken are the Faraday effect and magnetic circular dichroism in transmission and the magnetooptical Kerr effect in reflection. These phenomena have been studied in a large number of compounds and their macroscopical and microscopical properties are well understood [see, e.g., Refs. 1-3].

It has recently been shown that the combination of timereversal symmetry breaking with the absence of spaceinversion symmetry leads to new nonlinear magneto-optical effects. $^{4-6}$ During the last years, the magnetization-induced second harmonic generation (MSHG) has been successfully used for studies of magnetic surfaces, interfaces, and bulk properties of materials. $^{7-13}$ In this paper we show that the interaction of circularly polarized light with magnetized media leads to nonlinear magneto-optical phenomena that have no equivalence in linear magneto-optics. The key aspects of these phenomena will be analyzed and illustrated by SHG experiments in thin garnet films.

In the electric-dipole approximation, the second harmonic polarization $\mathbf{P}^{2 \omega}$ produced by a fundamental field $\mathbf{E}^{\omega}$ is described by where the polar tensor $\chi_{i j k}^{(2)}$ and the axial tensor $\chi_{i j k l}^{(3)}$ are only allowed in media with broken space-inversion operation $\overline{1}$.

Let us analyze the SHG produced by a circularly polarized excitation beam with an electric field $\mathbf{E}_{ \pm}^{\omega}=E_{0}\left(e_{X}\right.$ $\left.\pm i e_{Y}\right)$ in the longitudinal Faraday geometry in a magnetized thin film. The \pm signs refer to the right- $(+)$ and left- $(-)$ handed circular polarization of the fundamental light that propagates along the $z$ axis normal to the sample as shown in Fig. $1(\mathbf{k}\|\mathbf{M}\| Z \| z)$. $X Y Z$ is the laboratory coordinate system. The solution for the transmitted nonlinear radiation from a magnetized thin layer can be determined by solving Maxwell's equations with a nonlinear source polarization occupying a limited volume and then taking the limit as the thickness goes to zero. ${ }^{14}$ Then the induced SHG intensity can be written as

$$
\begin{aligned}
I^{2 \omega}\left(\mathbf{E}_{ \pm}^{\omega}, \mathbf{M}\right) \propto & E_{0}^{4}\left[\mid \chi_{x x x}-\chi_{x y y} \pm 2 i \chi_{x x y}+i\left(\chi_{x x x z}-\chi_{x y y z}\right) M_{z}\right. \\
& +\left.2 \chi_{x x y z} M_{z}\right|^{2}+\mid \chi_{y x x}-\chi_{y y y} \pm 2 i \chi_{y y x} \\
& \left.+i\left(\chi_{y x x z}-\chi_{y y y z}\right) M_{z}+\left.2 \chi_{y y x z} M_{z}\right|^{2}\right] .
\end{aligned}
$$

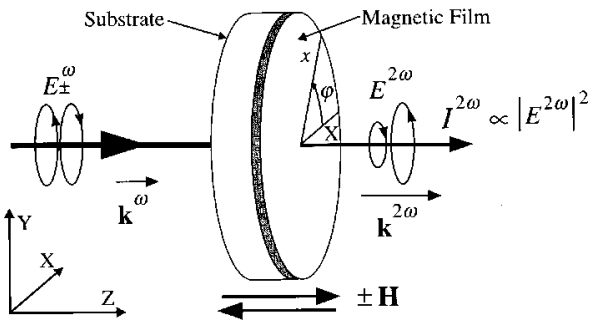

FIG. 1. Geometry of SHG measurements. The frame $x y z$ is related to the sample rotating with respect to the laboratory frame $X Y Z$ so that $z \| Z$. 
TABLE I. Nonlinear asymmetry of SHG intensity for different crystallographic classes in the geometry $\mathbf{k}\|\mathbf{M}\| z$ in transparency ( $t$ ) and absorption (a) region of crystals. $z$ is parallel to the axis of the highest symmetry.

\begin{tabular}{ccc}
\hline \hline & \multicolumn{2}{c}{$\begin{array}{c}\text { Nonlinear asymmetry } \\
\text { due to } \\
\text { Point } \\
\text { groups }\end{array}$} \\
$\chi_{i j k}^{(2)}$ or $\chi_{i j k l}^{(3)}$ & $\chi_{i j k}^{(2)}$ and $\chi_{i j k l}^{(3)}$ \\
\hline $1, m$ & elliptical (a) & elliptical (t, a) \\
$3, \overline{6}$ & circular (a) & circular (t, a) \\
$32,3 m, \overline{6} m 2$ & & circular (t, a) \\
\hline \hline
\end{tabular}

We define the nonlinear optical asymmetry due to the change of the fundamental light helicity as

$$
\rho_{h}=\frac{I^{2 \omega}\left(\mathbf{E}_{+}^{\omega}, \mathbf{M}\right)-I^{2 \omega}\left(\mathbf{E}_{-}^{\omega}, \mathbf{M}\right)}{I^{2 \omega}\left(\mathbf{E}_{+}^{\omega}, \mathbf{M}\right)+I^{2 \omega}\left(\mathbf{E}_{-}^{\omega}, \mathbf{M}\right)} .
$$

The nonlinear asymmetry due to the magnetization reversal is defined for each fundamental light helicity as

$$
\rho_{M}=\frac{I^{2 \omega}\left(\mathbf{E}^{\omega},+\mathbf{M}\right)-I^{2 \omega}\left(\mathbf{E}^{\omega},-\mathbf{M}\right)}{I^{2 \omega}\left(\mathbf{E}^{\omega},+\mathbf{M}\right)+I^{2 \omega}\left(\mathbf{E}^{\omega},-\mathbf{M}\right)} .
$$

It follows from Eqs. (3) and (4) that even in the absence of magnetization $M=0$, the SHG intensity may depend on the fundamental light helicity and the nonlinear asymmetry $\rho_{h}$ may have a nonzero value. Table I summarizes the crystallographic point groups (PG's) in which the nonlinear asymmetry $\rho_{h} \neq 0$ may arise for the fundamental light propagating along the $z$ axis. On the basis of symmetry analysis ${ }^{15}$ we find that the nonlinear asymmetry $\rho_{h}$ arises when $\chi_{x x x} \neq \chi_{y y y}$ $\neq 0$ and/or $\chi_{x x x z} \neq \chi_{y y y z} \neq 0$. In a pure form this occurs only in seven of the total number of 32 crystallographic classes. Note that fundamental light with circular polarization propagating along the threefold and the inversion sixfold axes gives rise to a circular SHG response because the rotational symmetry of the combined system (crystal and electromagnetic field) must be preserved. ${ }^{16}$ In this case the right-handed polarized fundamental light gives rise to left-handed SHG light and vice versa. For crystals of lower symmetry the SHG light becomes elliptical. The number of crystallographic classes permitting the nonlinear asymmetry increases for light propagating along an arbitrary direction with respect to the principal crystallographic axes and the resulting SHG light will in general have elliptical polarization.

Equations (2) and (3) show that the magnetizationinduced contribution to SHG is allowed in noncentrosymmetric crystals and that the nonlinear asymmetry may occur due to the interference of the crystallographic and magnetic parts of SHG. To demonstrate this, let us consider a crystal having crystallographic PG $32(x \| 2), 3 m$, or $\overline{6} m 2$ with a magnetization $\mathbf{M}$ along the optical axis. Well known examples of crystals with such symmetry are $\alpha$ quartz $\left(\alpha-\mathrm{SiO}_{2}, \mathrm{PG} 32\right)$ and lithium niobate $\left(\mathrm{LiNbO}_{3}, \mathrm{PG} 3 m\right)$, in which a magnetization $\mathbf{M}$ can be induced by an external magnetic field. Equation (3) gives the following expression for the SHG intensity:

$$
I^{2 \omega} \propto 8 E_{0}^{4}\left(\chi_{x x x}^{2}+\chi_{y y y z}^{2} M_{z}^{2} \pm 2 \chi_{x x x} \chi_{y y y z} M_{z}\right) .
$$

The interference term $\pm 2 \chi_{x x x} \chi_{y y y z} M_{z}$ gives rise to a nonzero value of the nonlinear asymmetry

$$
\rho_{h}=\rho_{M}=2 \chi_{x x x} \chi_{y y y z} M_{z} /\left(\chi_{x x x}^{2}+\chi_{y y y z}^{2} M_{z}^{2}\right) .
$$

This equation shows that in the case $\chi_{y y y z} M_{z} \ll \chi_{x x x}$, the induced asymmetry is a linear function of the magnetization. Its magnitude is defined by the ratio of magnetic to nonmagnetic contributions to $\mathbf{P}^{2 \omega}, \rho_{h}=\rho_{M} \simeq 2 \chi_{y y y z} M_{z} / \chi_{x x x}$. It must be emphasized that the nonlinear magneto-optical asymmetry is allowed even in the transparency region of crystals. This is in contrast to linear magneto-optics, where the effects of circular dichroism due to $\beta_{i j k}^{(2)}$ [see Eq. (1)] are allowed only in the absorption region of crystals. Here, by the absorption region we mean the linear absorption for both fundamental and SHG light, related to the imaginary part of the diagonal elements of $\chi^{(1)}$. This difference is directly related to the higher rank tensor for the nonlinear optical case. If the pump beam is linearly polarized with the wave vector k parallel to the threefold or sixfold axis, the SHG light becomes in general elliptically polarized with an ellipticity of the SHG light $\epsilon=-\arctan \left(\chi_{y y y z} M_{z} / \chi_{x x x}\right)$. In linear optics, linearly polarized light with $\mathbf{k}$ parallel to the optical axis always gives rise to linearly polarized outgoing light in lossless crystals.

The Onsager principle, which determines the symmetry of the kinetic coefficients in the presence of a magnetic field or spontaneous magnetization, states for the tensor of the dielectric susceptibility ${ }^{17}$

$$
\chi_{i j}^{(2)}(\mathbf{M})=\chi_{j i}^{(2)}(-\mathbf{M}) .
$$

In linear optics for the light propagating along optical axes this leads to a strict equivalence between the magnetization reversal and the change of the light helicity from left- to right-handed. In the nonlinear case, the magnetization reversal can be nonequivalent to the change of the incoming light helicity for the light propagating along the optical axis. For example, for crystals having crystallographic PG 3 or $\overline{6}$, Eq. (3) gives

$$
\begin{gathered}
I^{2 \omega} \propto 4 E_{0}^{4}\left[\left|\chi_{x x x} \mp i \chi_{y y y}+i \chi_{x x x z} M_{z} \pm \chi_{y y y z} M_{z}\right|^{2}\right. \\
\left.+\left|\chi_{y y y} \pm i \chi_{x x x}+i \chi_{y y y z} M_{z} \mp \chi_{x x x z} M_{z}\right|^{2}\right]
\end{gathered}
$$

and the equivalence does not hold for the absorption region of crystals where $\chi_{i j k}^{(2)}$ and $\chi_{i j k l}^{(3)}$ are complex. The nonequivalence arises due to the interference of time-invariant (crystallographic) and time-noninvariant (magnetic) contributions to the nonlinear polarization. The equivalence also breaks down for some crystals having lower or higher symmetry for the fundamental light propagating in an arbitrary direction, but in this case the SHG light will be elliptical.

To test some of these predictions for the nonlinear magneto-optical response under circular optical excitation magnetic garnet films, epitaxially grown on substrates with a controllable lattice mismatch, are suitable model systems. In these thin films, the original bulk space-inversion symmetry is broken as demonstrated by the observation of linear magneto-electric effects ${ }^{18}$ and SHG. ${ }^{10}$ It appears that the PG symmetry of the film is defined both by the substrate 

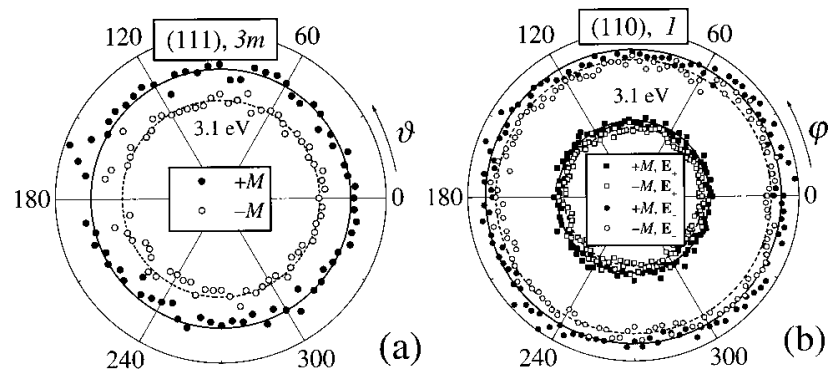

FIG. 2. (a) The SHG intensity for the garnet film on (111) substrate with symmetry $3 \mathrm{~m}$ as a function of the azimuthal analyzer angle $\vartheta$ for two opposite directions of the magnetization. (b) The SHG intensity in the (110) film of symmetry 1 for two opposite directions of the magnetization and light helicities as a function of the azimuthal sample angle $\varphi$.

orientation and by the growth mode. Thus, the symmetry $3 \mathrm{~m}$ is realized in films grown on a (111) oriented substrate, (210) films clearly show symmetry $m$, whereas strongly distorted (110) films are an example of the very low symmetry with the PG 1. The substrates of $\mathrm{Gd}_{3} \mathrm{Ga}_{5} \mathrm{O}_{12}$ garnet (GGG) as well as substituted GGG are transparent at the fundamental and SHG light and did not show any SHG signals. Garnet films of three types were studied in the work: (111) film with composition $(\mathrm{YLuBi})_{3}(\mathrm{FeGa})_{5} \mathrm{O}_{12}$, thickness $1 \mu \mathrm{m}$ and lattice misfit $-0.06 \%$; (210) film- $(\mathrm{YPrLuBi})_{3}(\mathrm{FeGa})_{5} \mathrm{O}_{12}, 10$ $\mu \mathrm{m}, 0.39 \%$; (110) film- $(\mathrm{YEuLuGa})_{3}(\mathrm{FeGa})_{5} \mathrm{O}_{12}, 10 \mu \mathrm{m}$, $0.2 \%$.

The experiments were done in transmission in the Faraday geometry at normal incidence with the light propagating along the $z$ axis (see Fig. 1) and with a magnetic field up to $H \simeq 0.23 \mathrm{~T}$ applied along $z$. In this geometry, Faraday rotation of the pump beam should be taken into account, whereas it can be ignored at the SHG frequency due to the very small effective thickness of the backside layer which serves as a source of the SHG light. Rotating the sample in the range $0 \leqslant \varphi \leqslant 360^{\circ}$ around the $z$ axis the SHG signals were registered for right- and left-handed input polarizations. In order to check the polarization state of the outgoing SHG light, the signals were registered also as a function of the azimuthal analyzer angle $\vartheta$.

The SHG signals were generated by the output of a modelocked Ti-sapphire laser working at a repetition frequency of $82 \mathrm{MHz}$, a pulse width of about $100 \mathrm{fs}$ at an average power on the sample between 100 and $200 \mathrm{~mW}$. SHG was studied in the spectral range of $1.545-1.725 \mathrm{eV}$ for the fundamental light, where the linear absorption of magnetic garnet films is $10-20 \mathrm{~cm}^{-1}$ (Ref. 19) and the fundamental beam propagates through thin films with a thickness of $1-10 \mu \mathrm{m}$ without any noticeable attenuation. The linear absorption is much higher at the second harmonic frequency, $\alpha \simeq 10^{4}-10^{5} \mathrm{~cm}^{-1} \cdot{ }^{19}$ Therefore, in transmission experiments, the detected SHG signal originates only from a backside layer with a thickness less than $1 \mu \mathrm{m}$.

In Fig. 2(a) the SHG intensity is shown for the garnet film of symmetry $3 m$ on substrate (111) for two opposite directions of the magnetization $\mathbf{M}$ along the $z$ axis as a function of the azimuthal analyzer angle $\vartheta$. This dependence is isotropic, showing that indeed the SHG light is circularly polarized for circular excitation. Replacing the incoming light helicity by

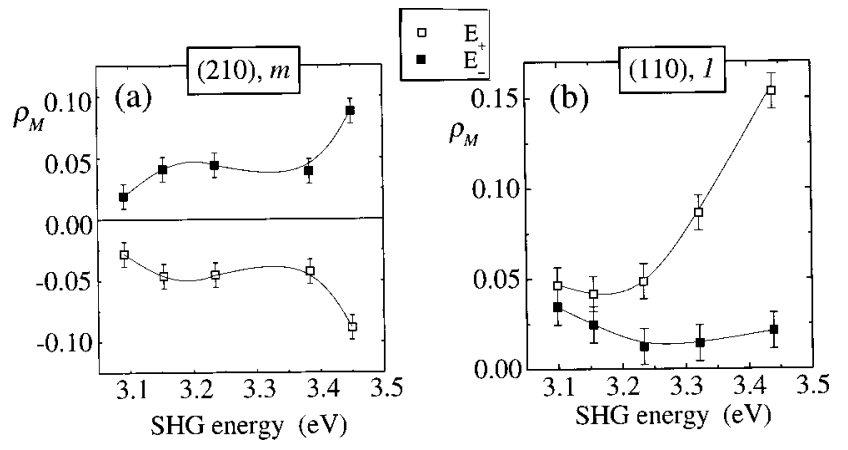

FIG. 3. Photon-energy dispersion of $\rho_{M}$ for the garnet film on a (210) substrate (a) and for the garnet film on a (110) substrate (b).

the opposite one leads to an interchange of the two dependencies for $\pm \mathbf{M}$. The nonlinear asymmetry arises due to the interference of magnetic and nonmagnetic parts of the nonlinear polarization $\mathbf{P}^{2 \omega}$. In the geometry $\mathbf{k}\|\mathbf{M}\| z$, the relevant components of $\chi_{i j k}^{(2)}$ are $-\chi_{x x x}=\chi_{y y x}=\chi_{y x y}=\chi_{x y y}$, the relevant components of $\chi_{i j k l}^{(3)}$ are $-\chi_{y y y z}=\chi_{y x x z}=\chi_{x x y z}$ $=\chi_{x y x z} \cdot{ }^{15}$ For crystals with the symmetry $3 m$ there is an equivalence between the magnetization reversal and the change of the light helicity in accordance with Eq. (6). Figure 2(b) shows the SHG intensity in the (110) film of symmetry 1 for two opposite directions of the magnetization and light helicities as a function of the azimuthal sample angle $\varphi$. In the geometry $\mathbf{k}\|\mathbf{M}\| z$, the relevant components of $\chi_{i j k}^{(2)}$ are $\chi_{x x x}, \chi_{y y y}, \chi_{y x x}, \chi_{x y y}, \chi_{x x y}=\chi_{x y x}, \chi_{y y x}=\chi_{y x y}$. The relevant components of $\chi_{i j k l}^{(3)}$ are $\chi_{x x x z}, \chi_{y y y z}, \chi_{y x x z}, \chi_{x y y z}, \chi_{x x y z}$ $=\chi_{x y x z}, \chi_{y y x z}=\chi_{y x y z}$. Four possible states of SHG intensity for this film have been observed in accordance with Eq. (3), i.e., in this case, the equivalence between magnetization reversal and changing the light helicity is broken.

According to Eqs. (3) and (6), the nonlinear asymmetry depends on both crystallographic and magnetic contributions and therefore should be sensitive to the splitting of electronic levels by the crystal field, spin-orbit coupling, and exchange interaction. The optical properties of iron garnets are due to relatively weak $d$ - $d$ transitions within $\mathrm{Fe}^{3+}$ ions above $1 \mathrm{eV}$ and much stronger transitions of the charge-transfer type above $2.8 \mathrm{eV} .{ }^{19}$ Obviously the latter are the major contributions to the nonlinear susceptibilities between 3.0 and 3.5 $\mathrm{eV}$.

Figure 3(a) shows the spectral variations of $\rho_{M}$ for the two light helicities in a film of symmetry $m$ on the substrate with orientation (210). Measurements have been done without analyzer in the geometry $\mathbf{k}\|\mathbf{M}\| z \|(210)$ and $x \|(001)$. For this geometry the relevant components of $\chi_{i j k}^{(2)}$ are $\chi_{y y y}, \chi_{y x x}, \chi_{x x y}=\chi_{x y x}$. The relevant components of $\chi_{i j k l}^{(3)}$ are $\chi_{x x x z}, \chi_{x y y z}, \chi_{y y x z}=\chi_{y x y z}$. The values of the nonlinear asymmetry $\rho_{M}$ have the same value and opposite sign for rightand left-handed polarized fundamental light. This supports the correspondence between the magnetization reversal and the change of the incoming light helicity.

Results for the nonlinear asymmetry $\rho_{M}$ in the garnet film of symmetry 1 on the substrate (110) are shown in Fig. 3(b). The values of $\rho_{M}$ for two different incoming light helicities have the same sign and thus the equivalence between the magnetization reversal and the light helicity change is broken. Introducing an analyzer behind the samples we checked 
the polarization state of SHG light and found that for (210) and (110) films the nonlinear asymmetry is elliptical, in contrast to the garnet film of symmetry $3 \mathrm{~m}$.

In conclusion, we have shown that right- and left-handed circular polarized excitation leads to different outputs of the second harmonic radiation in thin magnetic films. This kind of nonlinear optical asymmetry can be observed in lossless and absorbing media and appears to be directly related to the crystallographic symmetry as shown in Table I. The asymmetry arises due to an interference of nonlinear waves originating from the crystallographic and/or magnetic electric- dipole sources. In contrast to linear magneto-optics, we have observed a breaking of the correspondence between the magnetization reversal and the change of the light helicity in magnetic garnet films. The results appear in good agreement with the symmetry predictions.

Part of this work was supported by INTAS, the European TMR network (NOMOKE), the Russian Foundation for Basic Research, and the Program Laser Optics. V.V.P. and R.V.P. acknowledge the financial support of RIM during their stay at the Nijmegen University.
*Present address: FOM Institute for Plasma Physics, Edisonbaan 14, 3439 MN Nieuwegein, The Netherlands. Electronic address: kirilyuk@sci.kun.nl

${ }^{1}$ L. D. Barron, Molecular Light Scattering and Optical Activity (Cambridge University Press, Cambridge, 1982).

${ }^{2}$ J. Ferré, in Magnetism, Magnetic Materials and their Applications, edited by F. Leccabue and J. L. Sanchez Lamazares (IOP Publishing, Bristol, 1992), p. 167.

${ }^{3}$ A. K. Zvezdin and V. A. Kotov, Modern Magnetooptics and Magnetooptical Materials (IOP Publishing, Bristol, 1997).

${ }^{4}$ S. Kielich and R. Zawodny, Acta Phys. Pol. A 43, 579 (1973); S. Kielichand R. Zawodny, Opt. Acta 20, 867 (1973).

${ }^{5}$ Ru-Pin Pan, H. D. Wei, and Y. R. Shen, Phys. Rev. B 39, 1229 (1989).

${ }^{6}$ W. Hübner and K. H. Bennemann, Phys. Rev. B 40, 5973 (1989).

${ }^{7}$ B. Koopmans, M. Groot Koerkamp, Th. Rasing, and H. van den Berg, Phys. Rev. Lett. 74, 3692 (1995).

${ }^{8}$ M. Straub, R. Vollmer, and J. Kirschner, Phys. Rev. Lett. 77, 743 (1996).

${ }^{9}$ T. M. Crawford, C. T. Rogers, T. J. Silva, and Y. K. Kim, Appl. Phys. Lett. 68, 1573 (1996).
${ }^{10}$ V. V. Pavlov, R. V. Pisarev, A. Kirilyuk, and Th. Rasing, Phys. Rev. Lett. 78, 2004 (1997).

${ }^{11}$ Nonlinear Optics of Metals, edited by K. H. Bennemann (Oxford University Press, Oxford, 1998).

${ }^{12}$ V. V. Pavlov, G. Tessier, C. Malouin, P. Georges, A. Brun, D. Renard, P. Meyer, J. Ferré, and P. Beauvillain, Appl. Phys. Lett. 75, 190 (1999).

${ }^{13}$ M. Fiebig, D. Fröhlich, B. B. Krichevtsov, and R. V. Pisarev, Phys. Rev. Lett. 73, 2127 (1994).

${ }^{14}$ Here we restrict ourselves to the nonlinear optical polarization. The problem of propagation of the fundamental and SHG light will be discussed in a forthcoming paper.

${ }^{15}$ R. R. Birss, Symmetry and Magnetism (North-Holland, Amsterdam, 1966).

${ }^{16}$ H. J. Simon and N. Bloembergen, Phys. Rev. 171, 1104 (1968).

${ }^{17}$ L. D. Landau and E. M. Lifschitz, Electrodynamics of Continuous Media (Pergamon, New York, 1960).

${ }^{18}$ B. B. Krichevtsov, V. V. Pavlov, and R. V. Pisarev, Fiz. Tverd. Tela (Leningrad) 31, 77 (1989) [Sov. Phys. Solid State 31, 1142 (1989)].

${ }^{19}$ G. Winkler, Magnetic Garnets (Vieweg, Braunschweig, 1981). 